\title{
Opinion Dynamics via a Gossip Algorithm with Asynchronous Group Interactions
}

\author{
Emerico Aguilar and Yasumasa Fujisaki \\ Graduate School of Information Science and Technology, Osaka University \\ 1-5 Yamadaoka, Suita, Osaka 565-0871, Japan \\ E-mail: \{emerico-aguilar, fujisaki\}@ist.osaka-u.ac.jp
}

\begin{abstract}
Various models exist for representing the opinion formation process in social networks. The DeGroot consensus model, which is one of the earliest works in opinion dynamics, updates the opinions of the members of a group by taking into account their interrelationships and how much they value each other's opinions. In this model, the interactions and the weights given to opinions remain fixed during the entire duration of the process, which leads to a predictable behavior. As such, there are certain scenarios that can be better represented using other models. For asynchronous pairwise interactions, a gossip algorithm can be used as an alternative method for describing its opinion dynamics. This approach randomly selects the interacting pairs at every step of the algorithm. Thus, the opinion formation in this model is driven by a stochastic process. We propose to extend the pairwise gossip algorithm to accommodate asynchronous group interactions. We then analyze its behavior and compare it with the other models discussed in this paper.
\end{abstract}

\section{Introduction}

A social network is a collection of individuals and their relationships with one another. Different kinds of interactions occur within a social network which give rise to various social phenomena. The field of social network analysis deals with the examination of these interactions to look for patterns and to determine their effects on individuals and the network as whole.

An important topic in social network analysis is the opinion dynamics of social networks. Studies describing opinion propagation within groups of individuals began decades before the existence of massive communication platforms such as social media applications [1]-[3]. However, due to the increasing capacity of humans to connect with one another, understanding how opinions spread in social networks is becoming even more relevant.

DeGroot proposed one of the earliest models for describing the opinion formation process within a group of individuals [1]. This model, which we refer to as the consensus model in this paper, iteratively updates the opinions of indi- viduals as the weighted average of the opinions of the other individuals they are related to. The model assumes that relationships remain static throughout the duration of the process and the opinions are updated simultaneously during each time step. This denotes that interactions happen synchronously and the process is deterministic. This characteristic of the consensus model has motivated the development of other models that takes into consideration the fluidity of real-world interactions.

As an alternative to the consensus model, opinion propagation can be modeled using probabilistic asynchronous interactions. In [4], a gossip algorithm was used for modeling the opinion dynamics of random pairwise interactions.

In this paper, we extend the model in [4] by incorporating group interactions. We describe how it is related to the pairwise gossip algorithm and the consensus model, then we compare their behaviors via simulations.

The organization of this paper is as follows. Section 2 provides a review of the consensus model. Section 3 describes in detail the gossip algorithm for opinion dynamics. We introduce our proposed gossip algorithm with asynchronous group interactions in Section 4. We also compare its behavior with the other two models in this section. Finally, we mention our concluding remarks in Section 5.

\section{Consensus Model}

In the consensus model, a social network can be represented by a graph $G=(V, E)$ where the set of vertices $V=\{1, \ldots, n\}$ corresponds to the individuals or agents, and $E \subseteq V \times V$ is the set of edges such that $(i, j) \in E$ if and only if there is a relationship between agent $i$ and agent $j$. All agents adjacent to agent $i$ are called the neighbors of $i$, denoted by $N_{i}$. Edges may be directed or undirected depending on whether the relationships being represented are asymmetric or not. Since the gossip algorithm considered in [4] applies specifically to symmetric relationships, the scope of the discussion here is restricted to undirected graphs.

We now describe the opinion formation process of the consensus model. Let $x(k) \in \mathbb{R}^{n}$ be the opinions or states of the agents at time $k$. At each iteration of the model, the state of agent $i$ is updated as 


$$
x_{i}(k+1)=\sum_{j \in N_{i}} w_{i j} x_{j}(k)
$$

where $w_{i j}$ is the weight given by agent $i$ to the opinion of agent $j$. Let $W$ be the weight matrix where $w_{i j} \geq 0$ and $\sum_{j} w_{i j}=1$. The dynamics of the consensus model can then be written as

$$
x(k+1)=W x(k) .
$$

Let $L$ be the weighted Laplacian matrix of $G$ such that

$$
l_{i j}=\left\{\begin{array}{rr}
\sum_{j=1}^{n} a_{i j}, & i=j \\
-a_{i j}, & i \neq j
\end{array}\right.
$$

where $A$ is the weighted adjacency matrix of $G$. Then matrix $W$ can also be expressed as

$$
W=I-L
$$

The consensus model (1) is guaranteed to converge if the given graph is connected and aperiodic [1]. Additionally, if the model is applied to undirected graphs, $W$ is symmetric and doubly stochastic, which means it has a limiting distribution of $\mathbf{1}^{T} / n$, where $\mathbf{1}$ is the vector whose elements are all 1 and $y^{T}$ denotes the transpose of $y$. Therefore, when the previous properties hold, (1) converges almost surely to a consensus, as described by

$$
\lim _{k \rightarrow \infty} x(k)=\frac{\mathbf{1 1}^{T}}{n} x(0) .
$$

\section{Gossip Algorithm}

The gossip algorithm for modeling opinion formation is based on the randomized averaging algorithm on gossip networks [5]. This model describes the opinion dynamics when only a pair of agents can interact at a given time and it was proposed as an alternative to the consensus model when representing personal communications.

The algorithm can be described as follows. Consider a connected graph $G$ representing a group of agents and their relationships. At each time $k$, a pair of agents are randomly selected and they exchange their states. An agent $i \in V$ is first chosen with probability $1 / n$, followed by agent $j \in N_{i}$ with probability $p_{i j}$. Then, their states are updated as

$$
x_{i}(k+1)=x_{j}(k+1)=\frac{x_{i}(k)+x_{j}(k)}{2} .
$$

The probabilities for choosing a neighbor of agent $i$ given by the stochastic matrix $P$, where each $p_{i j}>0$ if and only if $(i, j) \in E$ and $\sum_{j} p_{i j}=1$ for all $i \in V$. Since any interacting pair of agents can exchange states, it implies that $G$ is an undirected graph. There are also no self-loops in $G$.

Let matrix $W(k)$ be a random matrix whose entries are dependent on the selected pair of agents $i$ and $j$ at time $k$. $W(k)$ can then be defined as

$$
W(k)=W_{i j}=I-\frac{\left(e_{i}-e_{j}\right)\left(e_{i}-e_{j}\right)^{T}}{2}
$$

where $e_{i}$ is a unit vector whose $i$ th element is one while the rest are zero. Then, the dynamics for the gossip algorithm is

$$
x(k+1)=W(k) x(k)
$$

which is a time-varying version of (1).

As shown in [4], the gossip algorithm converges almost surely to a consensus value of $\mathbf{1 1}^{T} x(0) / n$.

\section{Gossip Algorithm with Group Interactions}

As mentioned in the previous section, the gossip algorithm in [4] is restricted to pairwise interactions. Our work extends the gossip algorithm to allow asynchronous group interactions. Since this algorithm is an extension of the model described in [4], we also assume that the given graph is undirected and connected.

We now describe our proposed algorithm. Similar to the pairwise gossip algorithm, an agent $i$ is randomly selected per time step. However, instead of randomly selecting a neighbor, agent $i$ exchanges states with all its neighbors. Then, the states of agent $i$ and all agent $j \in N_{i}$ are simultaneously updated as

$$
\begin{gathered}
x_{i}(k+1)=\frac{1}{2} x_{i}(k)+\frac{1}{2} \sum_{j \in N_{i}} p_{i j} x_{j}(k) \\
x_{j}(k+1)=\frac{p_{i j}}{2} x_{i}(k)+\left(1-\frac{p_{i j}}{2}\right) x_{j}(k) .
\end{gathered}
$$

The random $W(k)$ matrix for the group gossip algorithm is based on the selected agent $i$ at time $k$, and can be written as

$$
W(k)=W_{i}=I-\sum_{j \in N_{i}} r_{i j}\left(e_{i}-e_{j}\right)\left(e_{i}-e_{j}\right)^{T}
$$

where $r_{i j}=p_{i j} / 2$. Using the $W(k)$ in (9), the dynamics of this extension of the gossip algorithm can be expressed similarly as (6).

We note that the sequence of matrices generated by $W(k)$ are independent and identically distributed, and each $W_{i}$ is doubly stochastic and has a positive diagonal. Also, since $\mathbb{E}\left[W_{i}\right]=\frac{1}{n} \sum_{i} W_{i}$, then the graph induced by $\mathbb{E}\left[W_{i}\right]$ is also connected. Therefore, based on the proofs in [6], 
$x(k)$ converges almost surely to a consensus value of $\mathbf{1 1}^{T} x(0) / n$. This also means that the consensus model, the pairwise gossip algorithm, and the group gossip algorithm converges to the same consensus value under similar conditions.

\subsection{Relationship with Other Models}

First, we describe the relationship of the group gossip algorithm with the pairwise gossip algorithm. If only one neighbor is picked in the group gossip algorithm and a probability of 1 is assigned to $p_{i j}$, then the matrix $W(k)$ will be the same for both variations of the gossip algorithm. This can be directly demonstrated by

$$
\begin{aligned}
W_{i} & =I-\sum_{j \in N_{i}} \frac{p_{i j}}{2}\left(e_{i}-e_{j}\right)\left(e_{i}-e_{j}\right)^{T} \\
& =\sum_{j \in N_{i}} p_{i j} I-\sum_{j \in N_{i}} p_{i j} W_{i j} \\
& =\sum_{j \in N_{i}} p_{i j} W_{i j}
\end{aligned}
$$

which shows that each iteration of the group gossip algorithm is the same as selecting all $j \in N_{i}$ in the pairwise gossip algorithm, and then computing the weighted average of their corresponding $W_{i j}$ matrices based on their probability of selecting each neighbor $j$.

Furthermore, based on the proofs in [4] and [5], we also note that

$$
\begin{aligned}
& \mathbb{E}[W(k)]=\sum_{i=1}^{n} \sum_{j=1}^{n} \frac{p_{i j}}{n} W_{i j} \\
& =I-\frac{1}{n} \sum_{i=1}^{n} \sum_{j \in N_{i}} \frac{p_{i j}}{2}\left(e_{i}-e_{j}\right)\left(e_{i}-e_{j}\right)^{T} \\
& =I-\frac{1}{2 n} \sum_{(i, j) \in E}\left(p_{i j}+p_{j i}\right)\left(e_{i}-e_{j}\right)\left(e_{i}-e_{j}\right)^{T} \\
& =I-L
\end{aligned}
$$

where $L$ is defined as

$$
L=\frac{1}{2 n}\left(\operatorname{diag}\left(\left(P+P^{T}\right) \mathbf{1}\right)-\left(\left(P+P^{T}\right)\right)\right) .
$$

From here, we can observe that $\mathbb{E}\left[W_{i}\right]=\mathbb{E}\left[W_{i j}\right]=I-L$, which coincides with (2). Thus, if we use $\mathbb{E}[W(k)]$ as the static weight matrix in (1), then the resulting dynamics will be similar to the consensus model.

We also highlight here the fact that, for all the three models, $x(k) \rightarrow \mathbf{1 1}^{T} x(0) / n$ as $k \rightarrow \infty$ when the given graph is undirected and connected, and $\mathbb{E}[W(k)]$ is used as the weight matrix for the consensus model. This means that, for the given conditions, their consensus value is the average of their initial states.

\subsection{Simulation Results}

We now compare the behavior of the three models considered in this paper using simulations. Let $V=\{1,2,3,4,5\}$ and $E=\{(1,2),(1,3),(1,4),(2,3),(3,5)\}$. Set $x(0)=$

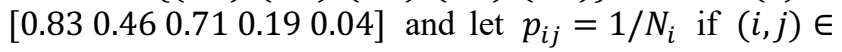
$E$, otherwise set it to zero. Figures $1-3$ show the results of the three models for the given values.

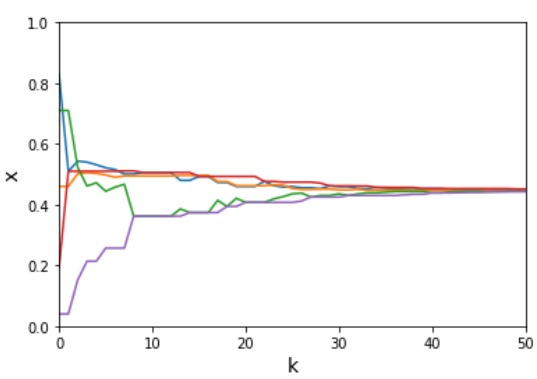

Figure 1: Pairwise gossip algorithm

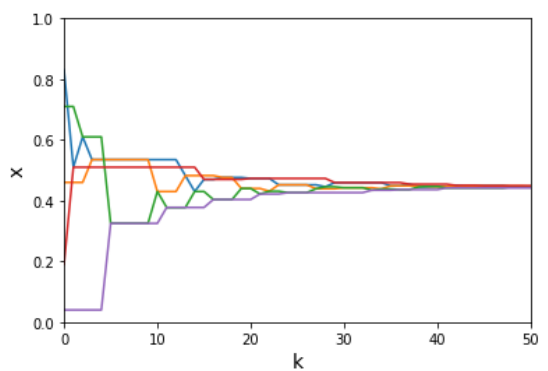

Figure 2: Group gossip algorithm

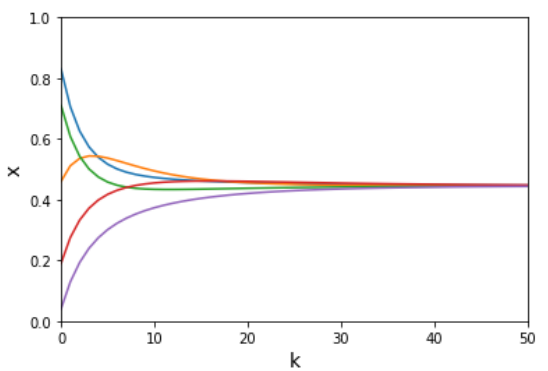

Figure 3: Consensus model

It can be seen that, while all three models converged to the same consensus, there is significant difference on how their states evolve from one time step to another. The pairwise gossip algorithm produces drastic changes in states, while the consensus model has smooth transitions. The behavior of the group gossip algorithm can be described as an intermediate between the other two models.

While the consensus model is deterministic, the interactions of the group gossip algorithm and the pairwise gossip algorithm are based on stochastic matrix processes. To further explore the distinction between both gossip algorithms, 
we compare the difference of their states and the states of the consensus model for each time step. We performed the comparisons on multiple Erdös-Rényi graphs with sizes of $n=10, n=50$, and $n=100$. For each of the three graph sizes, we computed the average difference on one hundred trials, with each trial having the same initial states for all the models. The initial states and the probability of creating edges on the Erdös-Rényi graphs are randomly assigned per trial. The results are shown in Figures $4-6$.

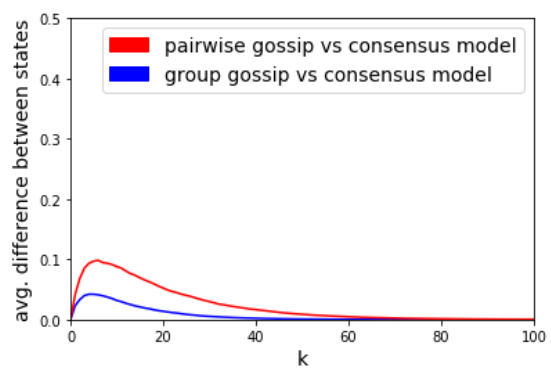

Figure 4: $n=10$

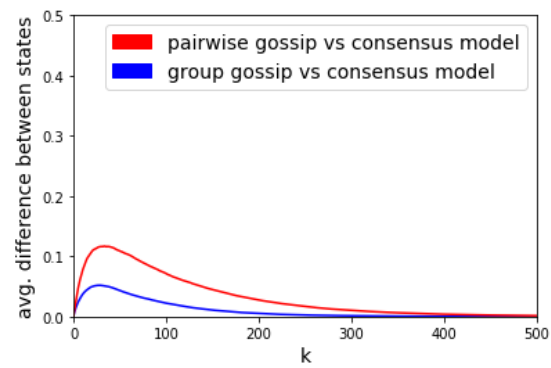

Figure 5: $n=50$

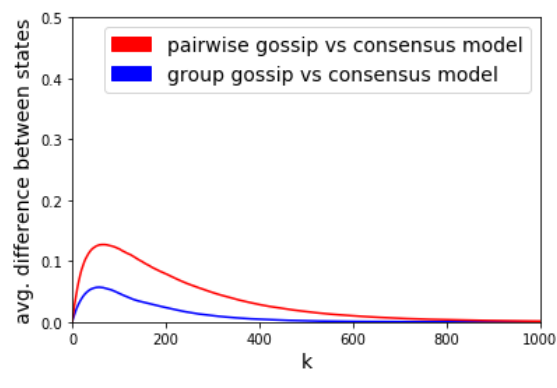

Figure 6: $n=100$

In all the graph sizes used in the simulation, the average difference of the pairwise gossip algorithm and the consensus model is significantly higher than the difference of the group gossip algorithm and the consensus model for smaller values of $k$. However, as the value of $k$ increases, the differences become less pronounced since the states approach the consensus value.

If the given conditions guarantee convergence for all the three models, then each of their updated states satisfy the inequality below at every iteration

$$
\min _{i} x_{i}(k) \leq x_{i}(k+1) \leq \max _{i} x_{i}(k) .
$$

In the consensus model, this implies that all states gradually approach the consensus value since they are all updated at each time $k$. However, only two states are updated at each time step of the pairwise group gossip algorithm. Thus, the average difference between $n-2$ states in the pairwise gossip algorithm and the consensus model continuously increases during their early phases, while the other two states are not guaranteed to have matching values as well. On the other hand, an average of $\frac{1}{n} \sum_{i}\left|N_{i}\right|+1$ states are updated per iteration of the group gossip algorithm, where $\left|N_{i}\right| \geq 1$. This means that, on the average, there are more states that approach the consensus value per iteration of the group gossip algorithm compared to the pairwise gossip algorithm. While the $\left|N_{i}\right|+1$ updated states are not guaranteed to match in the group gossip algorithm and the consensus model, the inequality in (10) also implies that gap between $\min _{i} x_{i}(k)$ and $\max _{i} x_{i}(k)$ is more likely to decrease at each iteration, which also reduces the possible differences between their $\left|N_{i}\right|+1$ updated states. This explains the patterns shown in Figures $4-6$.

\section{Conclusions}

In this work, we extended the pairwise gossip algorithm for modeling opinion dynamics to incorporate asynchronous group interactions. We also discussed how our proposed algorithm is related to the pairwise gossip algorithm and the consensus model, and then we compared the behaviors of the three models using simulations. Finally, we established that the evolution of the states in the group gossip algorithm is less drastic compared to the pairwise gossip algorithm during the early stages of their opinion formation process.

For future research, we plan to modify the group gossip algorithm such that a random subset of neighbors is selected per iteration. This can lead to an opinion dynamics model that has a wider range of applications.

\section{References}

[1] M. H. DeGroot: Reaching a consensus, Journal of the American Statistical Association, Vol. 69, No. 345, pp. 118-121, 1974.

[2] J. R. P. French: A formal theory of social power, Psychological Review, Vol. 63, pp. 181-194, 1956.

[3] K. Lehrer: Social consensus and rational agnoiology, Synthese, Vol. 31, pp. 141-160, 1975.

[4] L. T. H. Nguyen, T. Wada, I. Masubuchi, T. Asai, and Y. Fujisaki: Opinion formation over signed gossip networks, SICE Journal of Control, Measurement, and System Integration, Vol. 10, No. 3, pp. 266-273, 2017.

[5] S. Boyd, A. Ghosh, B. Prabhakar, and D. Shah: Randomized gossip algorithms, IEEE Transactions on Information Theory, Vol. 52, No. 6, pp. 2508-2530, 2006.

[6] A. Tahbaz-Salehi and A. Jadbabaie: Consensus over ergodic stationary graph processes, IEEE Transactions on Automatic Control, Vol. 55, No. 1, pp. 225-230, 2010. 